\title{
Cobalt hexammine induced tautomeric shift in Z-DNA: the structure of $d($ (CGCGCA).d(TGCGCG) in two crystal forms
}

\author{
S. Thiyagarajan, S. S. Rajan and N. Gautham* \\ Department of Crystallography and Biophysics, University of Madras, Guindy Campus, Chennai 600 025, India
}

Received September 4, 2004; Revised and Accepted October 14, 2004

PDB accession nos $1 \mathrm{XA} 2$ and 1XAM

\begin{abstract}
We report here the crystal structure of the DNA hexamer duplex d(CGCGCA).d(TGCGCG) at 1.71 A resolution. The crystals, in orthorhombic space group, were grown in the presence of cobalt hexammine, a known inducer of the left-handed Z form of DNA. The interaction of this ion with the DNA helix results in a change of the adenine base from the common amino tautomeric form to the imino tautomer. Consequently the $A: T$ base pair is disrupted from the normal Watson-Crick base pairing to a 'wobble' like base pairing. This change is accommodated easily within the helix, and the helical parameters are those expected for Z-DNA. When the cobalt hexammine concentration is decreased slightly in the crystallization conditions, the duplex crystallizes in a different, hexagonal space group, with two hexamer duplexes in the asymmetric unit. One of these is situated on a crystallographic 6-fold screw axis, leading to disorder. The tautomeric shift is not observed in this space group. We show that the change in inter-helix interactions that lead to the two different space groups probably arise from the small decrease in ion concentration, and consequently disordered positions for the ion.
\end{abstract}

\section{INTRODUCTION}

Metal ion interactions in nucleic acids have been widely studied $(1,2)$. In general, there are two views of cation binding that represent the vast majority of nucleic acid-cation interactions. One is the counter ion condensation theory that gives a picture of DNA in solution surrounded by a diffuse cloud of cations (3). These cations are considered to be delocalized along the length of the polymer and free from direct contacts with the DNA (i.e. they remain fully hydrated). The other is the view of tightly bound and partially dehydrated cations. Such interactions are observed in RNA structures, e.g. the four to six divalent cations that stabilize tRNA tertiary structure (4).
Within the past few years, it has become clear that the nature of cation binding to duplex DNA in most cases actually lies between these two extremes (2).

The interaction of cobalt hexammine with DNA is of particular interest because this ion strongly induces a transition from right-handed B type to left-handed Z type DNA (5-8). The transition between the $\mathrm{B}$ and $\mathrm{Z}$ forms takes place when the total free energy difference between the two forms is zero, with the ionic free energy favouring the $\mathrm{Z}$ form (9-11). Cobalt hexammine is five orders of magnitude more effective that sodium ions, and four orders of magnitude more effective than divalent magnesium ions in stabilizing the Z-DNA structure (5). Gessner et al. (12) explored the structural basis of this specific stabilizing ability. They found that the surface of Z-DNA provides an environment where the ion can form five hydrogen-bonding interactions with the DNA. This was in contrast to B-DNA, which does not have a comparable surface. To explain the greater effectiveness of cobalt hexammine compared to sodium and magnesium, it was suggested that the higher stability of the cobalt hexammine complex, compared to the magnesium-water complex, was due to the absence in the former of constant interchange of water molecules between those coordinating to the ion and the bulk waters. However, hexammine ions are also known to stabilize Z-DNA by water-mediated binding to the phosphate groups (13) and by non-specific interactions with DNA (14).

In our laboratory, we have a programme to use X-ray crystallography to study the effects of A:T base pairs in Z-DNA. We have undertaken the determination of the structures of a series of hexameric DNA fragments with sequences based upon the 'canonical' Z-DNA sequence d(CGCGCG $)_{2}$. We have earlier reported the crystal structures of $\mathrm{d}$ (CACGCG).d(CGCGTG) and d(CGCACG).d(CGTGCG) (15) (henceforth referred to as HA2 and HA4, respectively). The structures of HA2 and HA4 were studied in crystals grown in the presence of magnesium ions, though this ion could not be located in either of the structures. HA2 was also studied in crystals grown with ruthenium hexammine (14) replacing magnesium as the counter ion. This structure was virtually identical to the one with magnesium. In this structure too, the ion could not be located in the crystal, indicating a nonspecific role for it in stabilizing the left-handed structure. In this paper, we report the crystal structure of the hexanucleotide

*To whom correspondence should be addressed. Tel: +91 442230 0122; Fax: +91 442235 2494; Email: gautham@unom.ac.in or n_gautham@hotmail.com We dedicate this paper to the memory of late Prof. R. Srinivasan, former head of our department 
duplex d(CGCGCA).d(TGCGCG), i.e. HA6, in two crystal forms. The crystals were grown in the presence of cobalt hexammine at two slightly different concentrations, which lead to two different inter-helix interactions, and hence to two different space groups. Both the structures reported here show specific interactions of the hexammine ion with the atoms of the DNA bases.

\section{MATERIALS AND METHODS}

The component strands of the synthetic non selfcomplementary oligonucleotide duplex were purchased from M/s Microsynth, Switzerland and annealed to form the duplexes. Crystals were grown at room temperature (293 K) by the hanging drop vapour diffusion method. The crystallization drop contained $1 \mathrm{mM}$ of DNA in sodium cacodylate buffer at pH 6.9 together with $0.5 \mathrm{mM}$ cobalt hexammine chloride and $0.05 \mathrm{mM}$ spermine in the case of crystal 1 , and $0.3 \mathrm{mM}$ cobalt hexammine chloride and $0.05 \mathrm{mM}$ spermine in the case of crystal 2. The drop was equilibrated against 50\% methyl pentanediol in the reservoir. Two single crystals of size $0.15 \times 0.1 \times 0.1 \mathrm{~mm}$ were used for data collection on MAR-Research Imaging Plate systems, one at the National Area Detector Facility, Indian Institute of Science, Bangalore, India and the other at the GNR Laboratory for Structural Biology, Central Leather Research Institute, Chennai, India. The data set corresponding to crystal 1 was indexed in orthorhombic $\mathrm{P} 2{ }_{1} 2_{1} 2_{1}$ space group, while that corresponding to crystal 2 was indexed in hexagonal $\mathrm{P}_{5}$. Table 1 gives the data collection and refinement parameters. Crystal 1 diffracted to $1.71 \AA$ while crystal 2 diffracted to $2.0 \AA$. The structures were solved by molecular replacement using AMoRe (16) from the CCP4 suite (17). The starting model was based on the coordinates of fibre model Z-DNA (18) with A:T replacing one of the terminal $\mathrm{C}: \mathrm{G}$ base pairs.

In the orthorhombic data set, $8 \%$ of the data in the resolution range 18.1 to $1.71 \AA$ was flagged for cross validation, and the rest were used to refine the solution using REFMAC5 (19) of the CCP4 suite $(17)$. No $\sigma\left(F_{\mathrm{o}}\right)$ cut-off was applied. The structure fitted well into the $2 F_{\mathrm{o}}-F_{\mathrm{c}}$ electron density map after some minor changes. A total of 45 water molecules were added to the structure in cycles of refinement and map fitting. One strong density that appeared alone even at $4 \sigma$ level of the electron density map was identified as cobalt hexammine ion. The density was sufficient to locate four of the six

Table 1. Data collection and refinement details

\begin{tabular}{lll}
\hline Space group & $\mathrm{P} 2_{1}{ }_{1} 2_{1}$ & $\mathrm{P} 6_{5}$ \\
Resolution & $1.71 \AA$ & $2.0 \AA$ \\
Completeness (last shell) & $93.8 \%(99.1 \%)$ & $99 \%(100 \%)$ \\
$R$-merge (last shell) & $5.7 \%(14.9 \%)$ & $15 \%(29.8 \%)$ \\
Cell parameters & $17.98 \AA \AA$ & $35.59 \AA$ \\
& $30.93 \AA$ & $35.59 \AA$ \\
Number of reflections & $44.63 \AA$ & $44.52 \AA$ \\
Multiplicity & 2564 & $2495($ up to $1.86 \AA)$ \\
Mean I/sigma & 3.63 & 4.64 \\
$R$-factor & 5.8 & 2.4 \\
$R_{\text {free }}$ & $20.80 \%$ & $27.30 \%$ \\
\hline
\end{tabular}

amine groups around the cobalt atom. The other two amine groups were placed from geometrical considerations. The final $R$-factor was $20.8 \%\left(R_{\text {free }}=24 \%\right)$. There were no gross unexplained features in the map. The temperature factors of all the atoms of the structure, the water molecules and the cobalt hexammine ion were found to fall within acceptable ranges. The average thermal parameter was $\sim 22.4 \AA^{2}$ for all the atoms. Due to the high cylindrical symmetry in the DNA helix, at this stage there were still small uncertainties about the final model. Therefore, we verified the present structure to be the correct one by trying different models, which we had to reject. These attempts are described below, where relevant. The position of the cobalt ion was verified by an anomalous difference map (see Figure 2a). The peak height of the cobalt ion was $13.5 \mathrm{e}^{-3}$.

In the hexagonal system, the volume of the unit cell could accommodate more than one hexamer duplex in the asymmetric unit. AMoRe yielded the proper rotation and translation solution for one full hexamer. A few cycles of refinement and subsequent map calculation showed the extra electron density required to complete the structure. A dinucleotide was then built into this electron density. It became clear that the asymmetric unit contained one full hexamer plus a dinucleotide. The helix axis of the dinucleotide was positioned along the $6_{5}$ screw axis, thus generating an infinite disordered helix with the phosphate groups having partial occupancy. Because of the disorder, the dinucleotide step could stand for both $\mathrm{CpG} / \mathrm{CpG}$ as well as $\mathrm{CpA} / \mathrm{TpG}$. Therefore it was constructed as a TpG/ TpG step, in which the C5 methyl group of thymine was assigned occupancy $1 / 6$, and N2 of guanine was assigned occupancy 5/6. Thus each base pair in the helix stands onesixth of the time for an A:T base pair and five-sixths for a G:C base pair. The positions and occupancies of the disordered cobalt ion were fixed by trial and error, according to which values had the best effect on the map and the $R$-factors. The anomalous difference map could not be interpreted in this case as it had too much noise. The refinement converged at an $R$-factor of $27.3 \%$ with a free $R$-value of $35 \%$. There was some residual density in the map, which we were unable to interpret as part of the model. The final model is thus probably not free of errors, but these may not be germane to the discussion in this paper. In the rest of the paper, the orthorhombic structure is called OrthHA6, and has the following numbering scheme:

$5^{\prime} \mathrm{C} 1$ p G2 p C3 p G4 p C5 p A6 3'
$3^{\prime}$ G12 p C11 p G10 p C9 p G8 p T7 5'.

In the hexagonal structure, the fully ordered hexamer is referred to as HexHA6, while the hexamer built from the dinucleotide is called DinuHA6. The numbering scheme for HexHA6 is the same as above. The dinucleotide residues are given the numbers:

$5^{\prime}$ p T13 p G14 3'

$3^{\prime}$ p G16 p T15 5'.

G14 and G16 will be addressed as A14 and A16 respectively, whenever these purines are considered to represent adenine bases. A similar numbering scheme is adopted for other hexameric sequences taken for comparison. Conformational and helical parameter calculations were carried out using the 
programs FREEHELIX (20) and CURVES (21). The coordinates and structure factors have been deposited in the NDB (code ZD0013 and ZD0014).

\section{RESULTS AND DISCUSSION}

There are three independent Z-DNA duplexes (OrthHA6, HexHA6 and DinuHA6) in the two crystal structures. Overall, the structure of the duplexes in both space groups is left handed Z-type DNA. The purines and pyrimidines of the structure take alternate syn and anti conformations respectively, about their glycosidic bond. The sugar puckering follows the pattern seen in other similar crystal structures. The base stacking patterns show no significant difference from what is expected in Z-DNA helices (22). Table 2 compares the three helices in the two structures with each other, as well with the fibre model of left handed Z-DNA (18), which was derived from the crystal structure of $d(C G)_{3}$. (Though the helix DinuHA6 exists in the crystal as a disordered, quasicontinuous, infinite helix, we have chosen an arbitrary hexamer to represent it.) Clearly the helices OrthHA6, HexHA6 and DinuHA6 are all closely similar to the fibre model. This is despite the different packing environments.

The orthorhombic crystal has one independent duplex. In this packing mode, the hexamer duplexes are arranged one over the other by crystallographic symmetry to form infinite helical columns. The single A:T base pair breaks the 2-fold sequence symmetry of the hexamer. Consequently, the helix axis may be considered to have a direction, and may be represented by an arrow. Then, looking down the $c$-axis, as we go along the $a$-axis, all the adjacent columns point in the same direction, while along $b$-axis the helices in adjacent columns are oriented in opposite directions (Figure 1a). The hexagonal crystal has two independent duplexes. One of them is a hexamer, and is located at a general position in the unit cell. Here again, symmetry related hexamers stack one over the other to form infinite helical columns. All these columns are oriented in the same direction (Figure 1b). The other duplex is placed on the crystallographic 6-fold screw axis. The 6-fold screw symmetry of the $\mathrm{Z}$ type helix coincides with the crystallographic 6 -fold. The asymmetric unit is therefore a dinucleotide step, which is repeated infinitely to generate the helical column. Due to the missing phosphate groups for every six base pairs, as well as due to the presence of an A:T base pair in the sequence, this column of helices is disordered, and we cannot distinguish its direction. The disordered and ordered columns alternate along both $a$ - and $b$ - axes.

The most significant differences between the orthorhombic and hexagonal structures are in the ion binding modes. In the orthorhombic structure, one cobalt hexammine ion was clearly

Table 2. Root mean square deviation (in $\AA$ ) of the positions of common atoms in Z-DNA fibre model [d(CG) $)_{3}$, OrthHA6, HexHA6 and DinuHA6 after least squares superposition

\begin{tabular}{lllll}
\hline $\mathrm{d}(\mathrm{CG})_{3}$ fibre & 0.000 & & & \\
OrthHA6 & 0.720 & 0.000 & & \\
HexHA6 & 0.549 & 0.612 & 0.000 & \\
DinuHA6 & 0.546 & 0.562 & 0.475 & 0.000 \\
& $\mathrm{~d}(\mathrm{CG})_{3}$ fibre & OrthHA6 & HexHA6 & DinuHA6 \\
\hline
\end{tabular}

located from the Fourier map, as well as the anomalous difference map (Figure 2a). The amine groups of the cation form hydrogen bonds with the atoms of adenine 6 of one duplex (A), and the G12:C1 base pair of a symmetry-related duplex (B). The ion also interacts with guanine 4 of a third, symmetryrelated hexamer (Duplex C) (Figure 2a). Each ion thus binds to one adenine base, one cytosine base, and two guanine bases. Hydrogen bonding distances are given in Table 3.

Of these three sets of interactions, the one with the adenine is the strongest. There are four possible hydrogen bonds between the ion and adenine 6. Two amine groups make a pair of bifurcated hydrogen bonds with N7. The N6 amine group of adenine makes another pair of bifurcated hydrogen bonds with the amine groups of the ion (Figure 2b). Earlier work on metal ion interactions with A:T base pairs (23) has shown that the approach of any cation to O4 oxygen of thymine leads to destabilization of the base pair. Also, the adenine base has a weaker dipole moment than guanine, and this favours the metal ion interaction with guanine rather than with adenine (24). In view of these, we checked the interaction by rebuilding the model with a different orientation for the helix, so that the terminal G:C base pair replaces the A:T base pair. The ion would now be bound to three guanine bases of adjacent helices. However the refinement in this case did not proceed smoothly. Both $R$ and $R_{\text {free }}$ increased by $>4 \%$. The electron density map also clearly indicated that the re-modelled G:C base pair was in fact an A:T base pair, confirming the position of the A:T base pair near the ion (Figure 2b). We also rechecked it by assuming that the crystals contained a duplex of only one sequence, viz. d(TGCGCG). In this case there would be two G:T wobble base pairs, one at each end of the helix. However this possibility also was ruled out by omit maps that showed clearly that the base pair interacting with the ion was indeed an A:T base pair. It has been shown that the pyramidalization of hydrogen atoms of N6 adenine induced by the interactions with the cation would destabilize the adenine-thymine base pairing (25). Destabilization, but no rupture, is apparent in the present structure. The geometry of the interaction and of the A:T base pair suggests that the cobalt hexammine ion has induced a tautomeric shift of the adenine base from the amino to the imino form. This has resulted in a higher shear and stagger of the $\mathrm{A}$ :T base pair. It has shear of $\sim 1.82 \AA$ and stagger of $\sim 0.25 \AA$. For comparison, shear and stagger at other base pairs are $\sim \pm 0.2$ and $\pm 0.05 \AA$, respectively. The shearing at A6:T7 induces a 'wobble' type base pairing scheme between A6 and T7, resembling a G:T wobble base pair (26-28). It has been earlier reported that the interaction of metal hexammines stabilizes G:T wobble base pairs, particularly in RNA. (29-31). A wobble-type A:T base pairing scheme is possible only when N1 of adenine 6 is protonated, and the N6 amino group becomes a hydrogen bond acceptor by taking the imino form. Thus the presence of the ion has induced a shift in adenine from the amino to the imino tautomeric form. The present structure is the first example of a tautomeric shift in adenine in an oligonucleotide, and the consequent wobble-type A:T base pair, induced by cobalt hexammine. It has been shown before that N6-methoxy adenosine exists as imino tautomer when the ribose sugar is tri-methylated at $2^{\prime}, 3^{\prime}$ and $5^{\prime}$ positions respectively (32). Also, in another study of the structure of the ternary complex of mercury with 1,3-dimethyl-uracil and 9-methyladenine, 
(a)

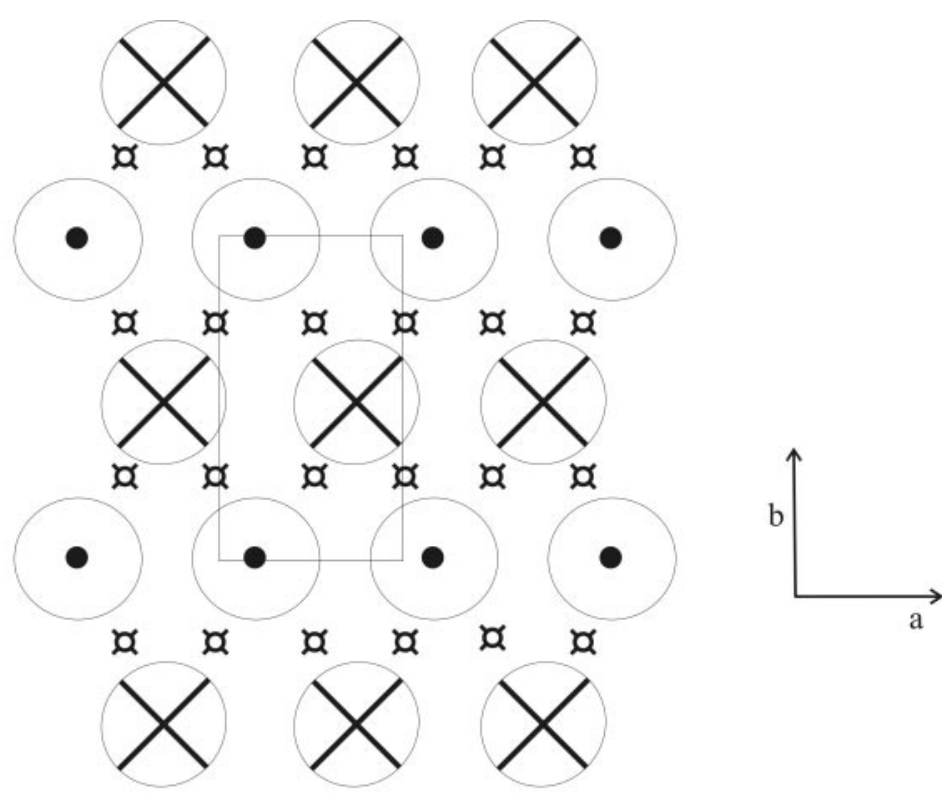

(b)
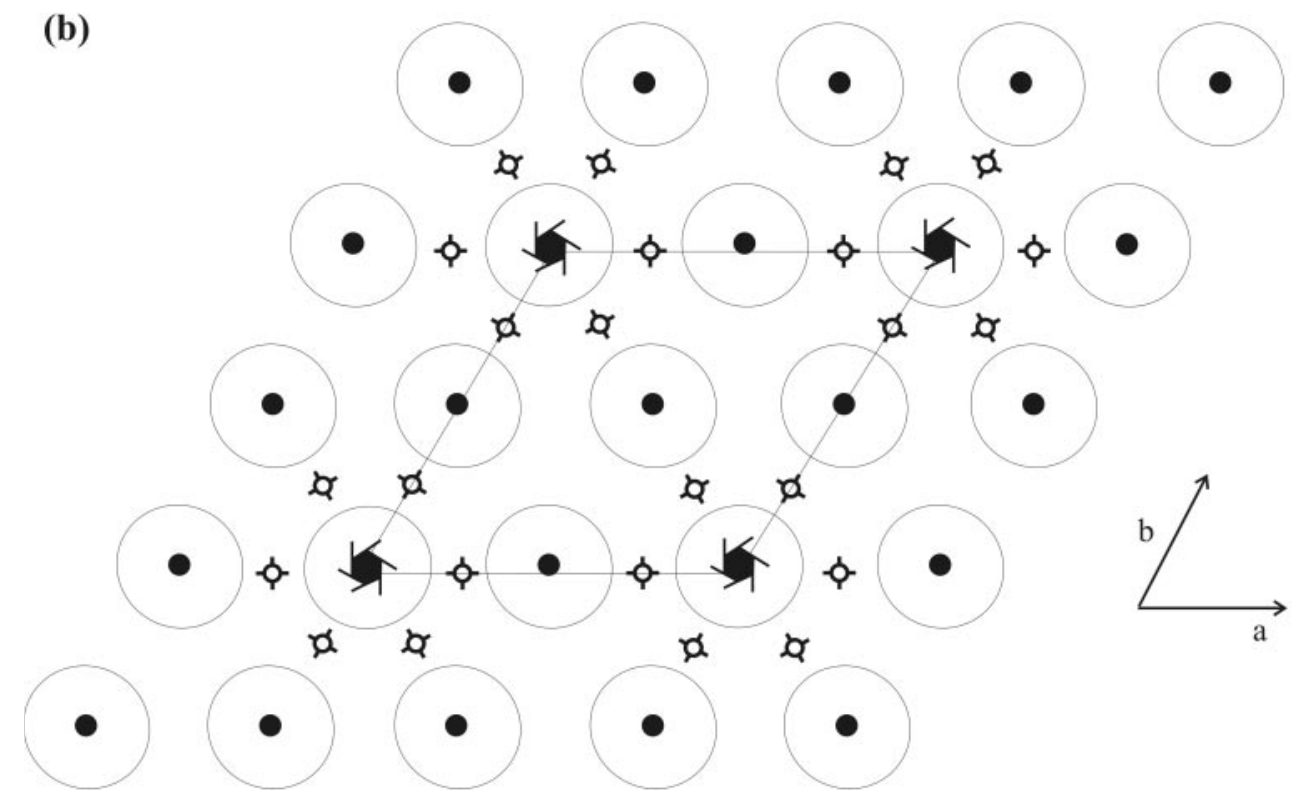

Q

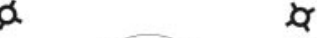

d. $\rightarrow$

Figure 1. Schematic representation of the packing of the helical columns in the two space groups. Circles with a thick dot in the centre represent columns of helices in one direction, while those with a cross represent columns of helices in opposite direction. Ions are represented by small stars. (a) Packing in the P2 $2_{1} 2_{1}$ structure. (b) Packing in the P6 5 structure. Circles with $6_{5}$ screw symbols inscribed indicate the disordered columns built from DinuHA6.

Zamora et al. (33) have shown that $\mathrm{Hg}^{2+}$ ions induce the imino tautomer of adenine, and this shift could favour mismatch base pairing between the imino adenine and cytosine. Other studies also have shown that such tautomeric transformations are often associated with mismatch base pairing leading to mutations during the process of translation or replication $(34,35)$.

The other two interactions made by the ion with the DNA primarily involve guanine bases. Usually, in metal-DNA interactions, metal ions bind to guanine through a coordination bond with $\mathrm{N} 7$ and $\mathrm{O} 6$ either by direct coordination or by water-mediated hydrogen bonds (12,36-38). In the interaction of the hexammine ions with the bases, the amine groups replace the water molecules. Thus in the interaction of cobalt hexammine with guanine, the amine groups usually make hydrogen bonds with both N7 and O6 of the base (12). In the present structure, the ion-guanine (G12) interaction however has only one hydrogen bond, and that is with O6 (Figure 3). The N7 of this base is exposed to the solvent, with the nearest water molecule at $3.64 \AA$ distance from N7. In this case, the cytosine base paired to G12 also interacts with the cobalt hexammine ion. The N4 group of cytosine interacts 
(a)
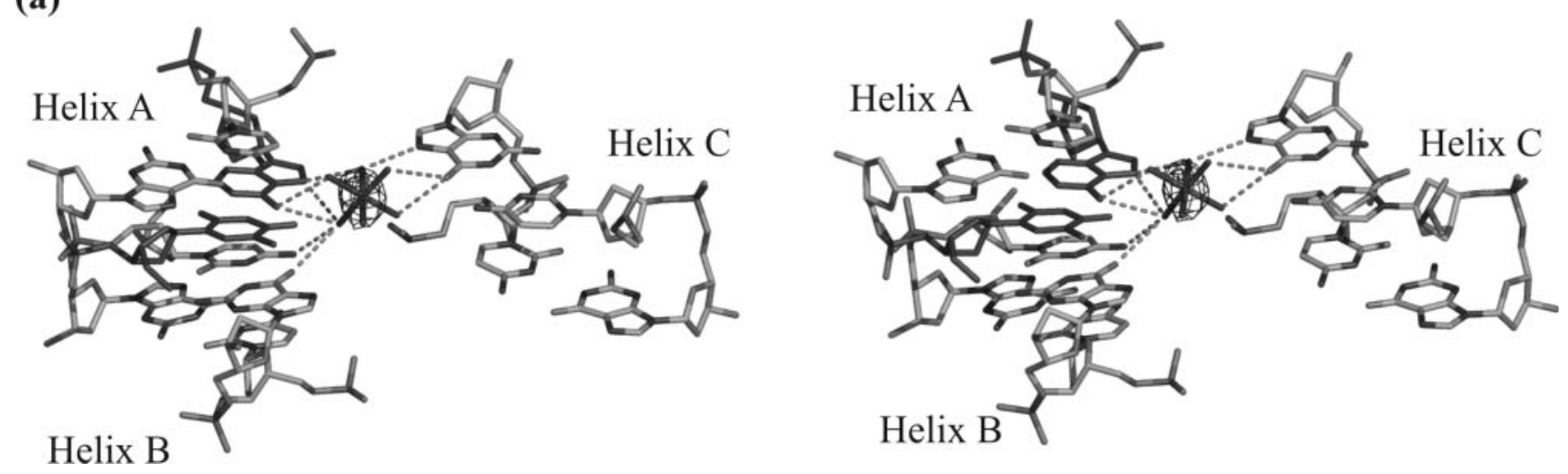

(b)

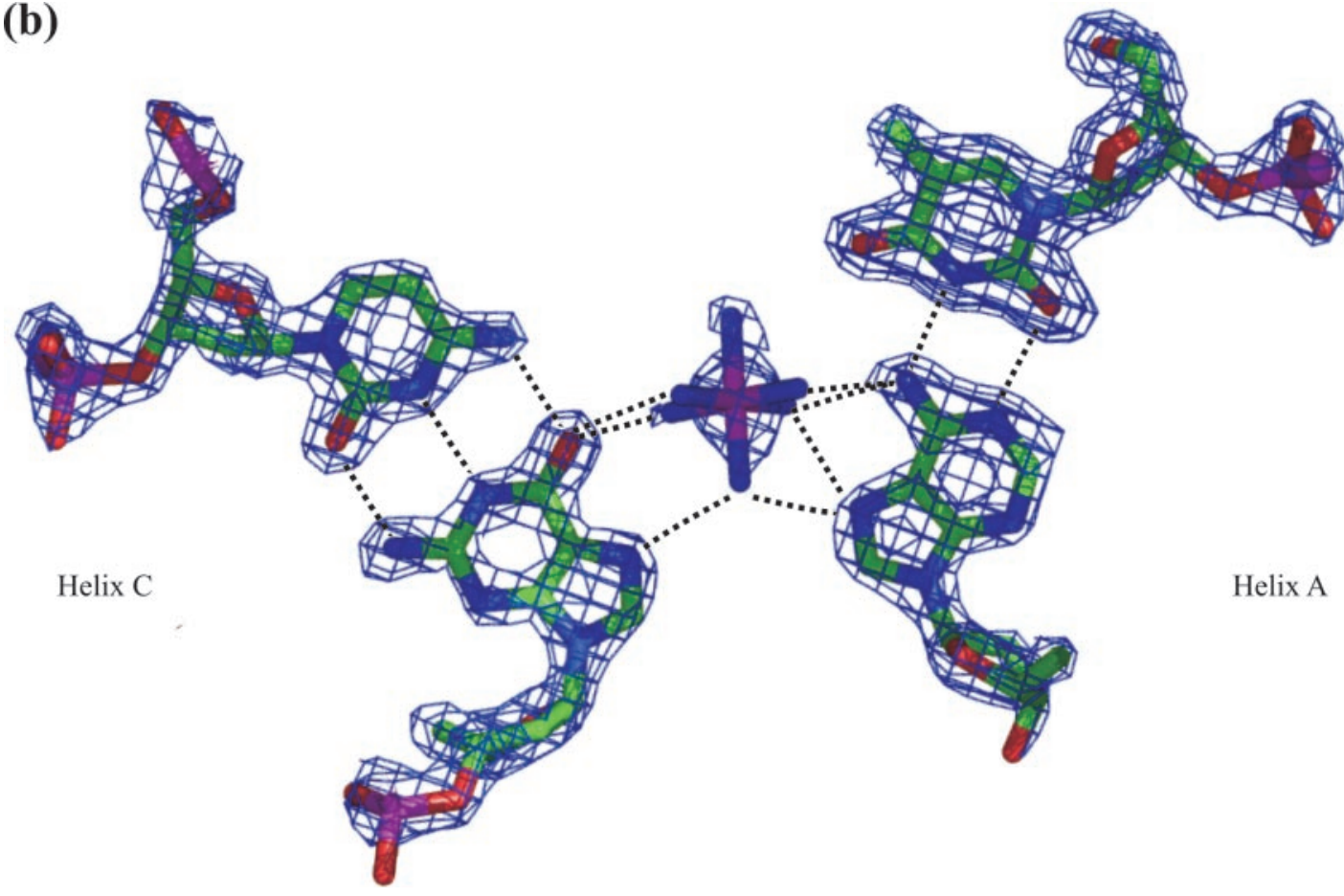

Figure 2. (a) Stereo view of ion binding mode in OrthHA6. Two base pairs from each of the three interacting helices are shown. The electron density contours shown for the cobalt ion were obtained from a map calculated using the anomalous differences in the structure factors as Fourier coefficients. The phases were calculated from the structure without the cobalt atom. (b) Ion interaction with helix A and helix C in OrthHA6 shown along with the $\left(2 F_{\mathrm{o}}-F_{\mathrm{c}}\right)$ electron density drawn at $1.2 \sigma$. The dotted lines represent hydrogen bonds.

with the amine group, which pulls the base towards the ion. This results in an increased shear of $-1.14 \AA$ in G12:C1 base pair, in contrast to $\pm 0.2 \AA$ base pair shear at other base pairs. This interaction disturbs the normal WatsonCrick base pairing scheme in G12:C1 base pair (Figure 3). The other guanine base (G4) interacts with the cobalt hexammine ion in the conventional pattern of having hydrogen bonds between the amine groups and N7 and O6 atoms of the base (Figure 3).

In the hexagonal structure there is a disordered cobalt hexammine ion in the asymmetric unit, with one-third occupancy. The pattern of interactions made by the ion with the neighbouring helices is different from the one in the orthorhombic crystal. As mentioned above, the crystal is made of alternate columns of ordered and disordered hexamers. The arrangement is such that each disordered column is surrounded by six ordered columns, while each ordered column is surrounded by two disordered columns and four ordered columns. The interaction of the ion with the disordered hexamer that forms part of a disordered column may be considered to be either at a $\mathrm{CpG}$ step, or at a $\mathrm{CpA}$ step. If at the $\mathrm{CpG}$ step, the ion is bound to $\mathrm{O} 6$ and $\mathrm{N} 7$ of $\mathrm{G} 14$, as well as to $\mathrm{O} 6$ of G16. If the interaction is at the $\mathrm{CpA}$ step, the ion makes hydrogen bonds with $\mathrm{O} 6$ and N7 of G14, as well as with N6 of A16. Since the direction of the DinuHA6 could not be distinctly specified, the above interactions could also be considered to be between the ion and N6 and N7 of A14 and O6 of G16. The partially occupied ion also makes hydrogen bond interactions with the G12 and A6 bases of the ordered hexamer. These interactions are shown in Figure 4. Again Table 3 gives details 
Table 3. Details of ion-DNA hydrogen bond geometries. Details of the cross-strand hydrogen bonds are also included

\begin{tabular}{|c|c|c|c|c|}
\hline Space group & Donor & Acceptor & Distance $(\AA)$ & Remarks \\
\hline \multicolumn{5}{|l|}{$\mathrm{P} 22_{1} 2_{1}$} \\
\hline & {$\left[\mathrm{Co}\left(\mathrm{NH}_{3}\right)_{6}\right]^{3+}(\mathrm{N} 6)$} & N7(A6) & 2.95 & \\
\hline & {$\left[\mathrm{Co}\left(\mathrm{NH}_{3}\right)_{6}\right]^{3+}(\mathrm{N} 3)$} & N7(A6) & 3.03 & \\
\hline & {$\left[\mathrm{Co}\left(\mathrm{NH}_{3}\right)_{6}\right]^{3+}(\mathrm{N} 3)$} & N6(A6) & 3.10 & \\
\hline & {$\left[\mathrm{Co}\left(\mathrm{NH}_{3}\right)_{6}\right]^{3+}(\mathrm{N} 3)$} & O6 (G12) & 3.13 & \\
\hline & {$\left[\mathrm{Co}\left(\mathrm{NH}_{3}\right)_{6}\right]^{3+}(\mathrm{N} 6)$} & O6 (G12) & 3.04 & \\
\hline & {$\left[\mathrm{Co}\left(\mathrm{NH}_{3}\right)_{6}\right]^{3+}(\mathrm{N} 3)$} & N4 (C1) & 3.19 & \\
\hline & {$\left[\mathrm{Co}\left(\mathrm{NH}_{3}\right)_{6}\right]^{3+}(\mathrm{N} 2)$} & O6(G4) & 2.86 & \\
\hline & {$\left[\mathrm{Co}\left(\mathrm{NH}_{3}\right)_{6}\right]^{3+}(\mathrm{N1})$} & O6(G4) & 3.01 & \\
\hline & {$\left[\mathrm{Co}\left(\mathrm{NH}_{3}\right)_{6}\right]^{3+}(\mathrm{N} 6)$} & N7(G4) & 3.06 & \\
\hline & O6 (G12) & N6 (A6) & 3.04 & Cross-strand hydrogen bond \\
\hline \multirow{10}{*}{$\mathrm{P}_{5}$} & {$\left[\mathrm{Co}\left(\mathrm{NH}_{2}\right)_{6}\right]^{3+}(\mathrm{N} 4)$} & O6 (G14) & 277 & Jon bound to disordered \\
\hline & {$\left[\begin{array}{lll}{[6(10113 / 6]} & (1,4)\end{array}\right.$} & $00(014)$ & 2.11 & DinuHA6. G14 could be A14 as well \\
\hline & {$\left[\mathrm{Co}\left(\mathrm{NH}_{3}\right)_{6}\right]^{++}(\mathrm{N} 4)$} & N7 (G14) & 3.15 & \\
\hline & {$\left[\mathrm{Co}\left(\mathrm{NH}_{3}\right)_{6}\right]^{3+}(\mathrm{N} 3)$} & O6 (G14) & 2.78 & \\
\hline & {$\left[\mathrm{Co}\left(\mathrm{NH}_{3}\right)_{6}\right]^{3+}(\mathrm{N} 3)$} & O6 (G16) & 3.04 & \\
\hline & {$\left[\mathrm{Co}\left(\mathrm{NH}_{3}\right)_{6}\right]^{3+}(\mathrm{N} 2)$} & O6(G12) & 2.96 & Ion bound to ordered HexHA6 \\
\hline & {$\left[\mathrm{Co}\left(\mathrm{NH}_{3}\right)_{6}\right]^{3+}(\mathrm{N} 1)$} & N7(G12) & 2.81 & \\
\hline & {$\left[\mathrm{Co}\left(\mathrm{NH}_{3}\right)_{6}\right]^{3+}(\mathrm{N} 5)$} & O6(G12) & 2.30 & \\
\hline & {$\left[\mathrm{Co}\left(\mathrm{NH}_{3}\right)_{6}\right]^{3+}(\mathrm{N} 5)$} & $\mathrm{N} 2(\mathrm{~A} 6)$ & 3.10 & \\
\hline & O6 (G12) & N6 (A6) & 3.05 & Cross-strand hydrogen bond \\
\hline
\end{tabular}

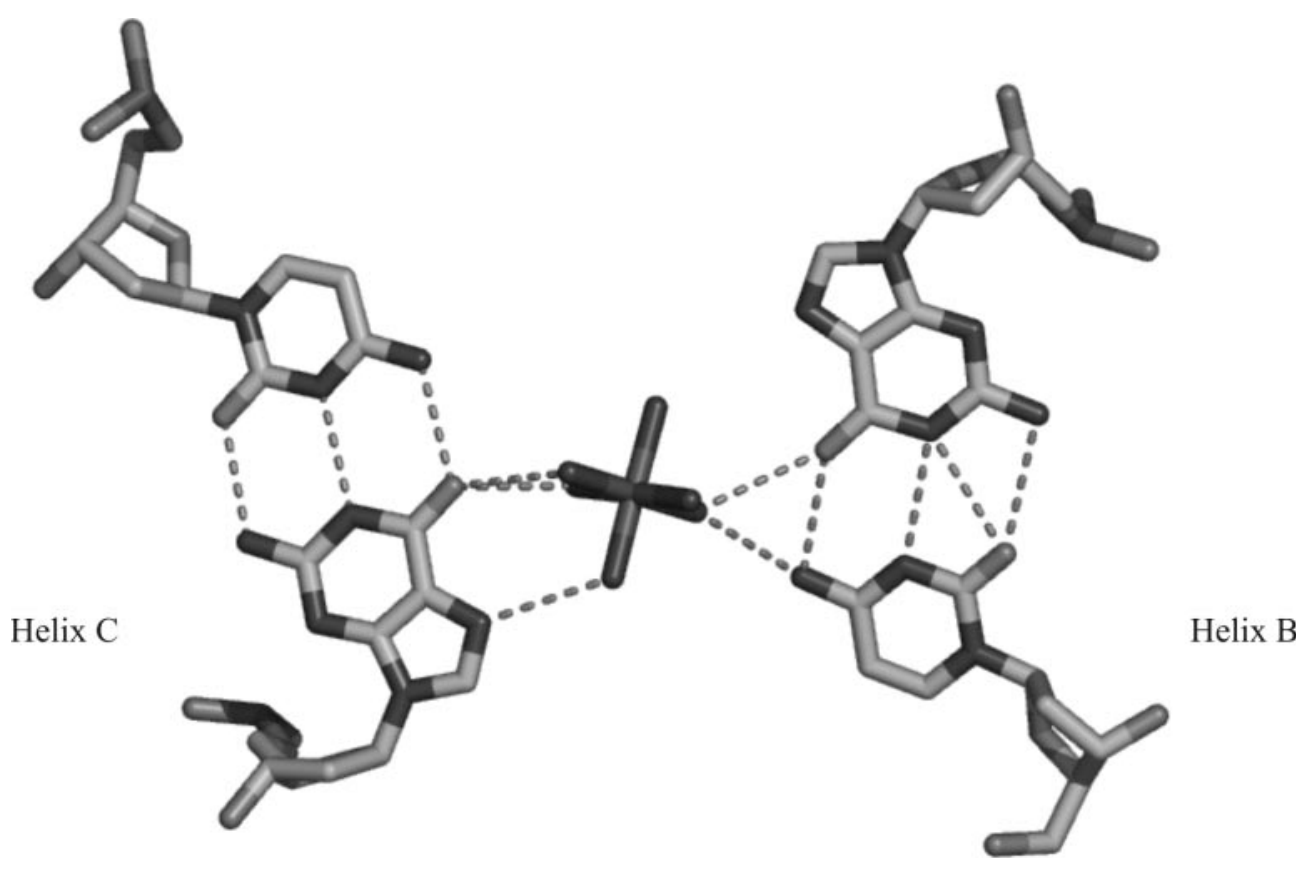

Figure 3. Details of the ion interaction geometry in OrthHA6. Interaction with G4 of helix C and the G12:C1 base pair of helix B. The dotted lines represent hydrogen bonds.

of the hydrogen bond geometries. Unlike the orthorhombic case, no particular change is seen in the base pairing scheme due to the ion interactions. Since these ion binding modes are so different as compared to the orthorhombic structure, efforts were made to identify G12 as A6. If this were so, the arrow representing the helix axis would point in the opposite direction. But $R$-factors as well as electron density maps confirmed that the ion was indeed bound to G12.

The differences in the ion binding patterns, and the consequent differences in helical packing and crystallographic space groups of the two crystal forms, probably arise from differences in the cobalt hexammine concentrations used in the two crystallization conditions.

This may also explain why there are two types of helices in the hexagonal structure. In the orthorhombic crystal, one unit cell has four cobalt hexammine ions. In the hexagonal crystal, one unit cell has only two cobalt hexammine ions despite the fact that the cell volume has doubled. As shown in Figure 1, in the orthorhombic crystal the adjacent rows of helical columns along the $b$-axis are aligned in opposite directions. The cobalt 

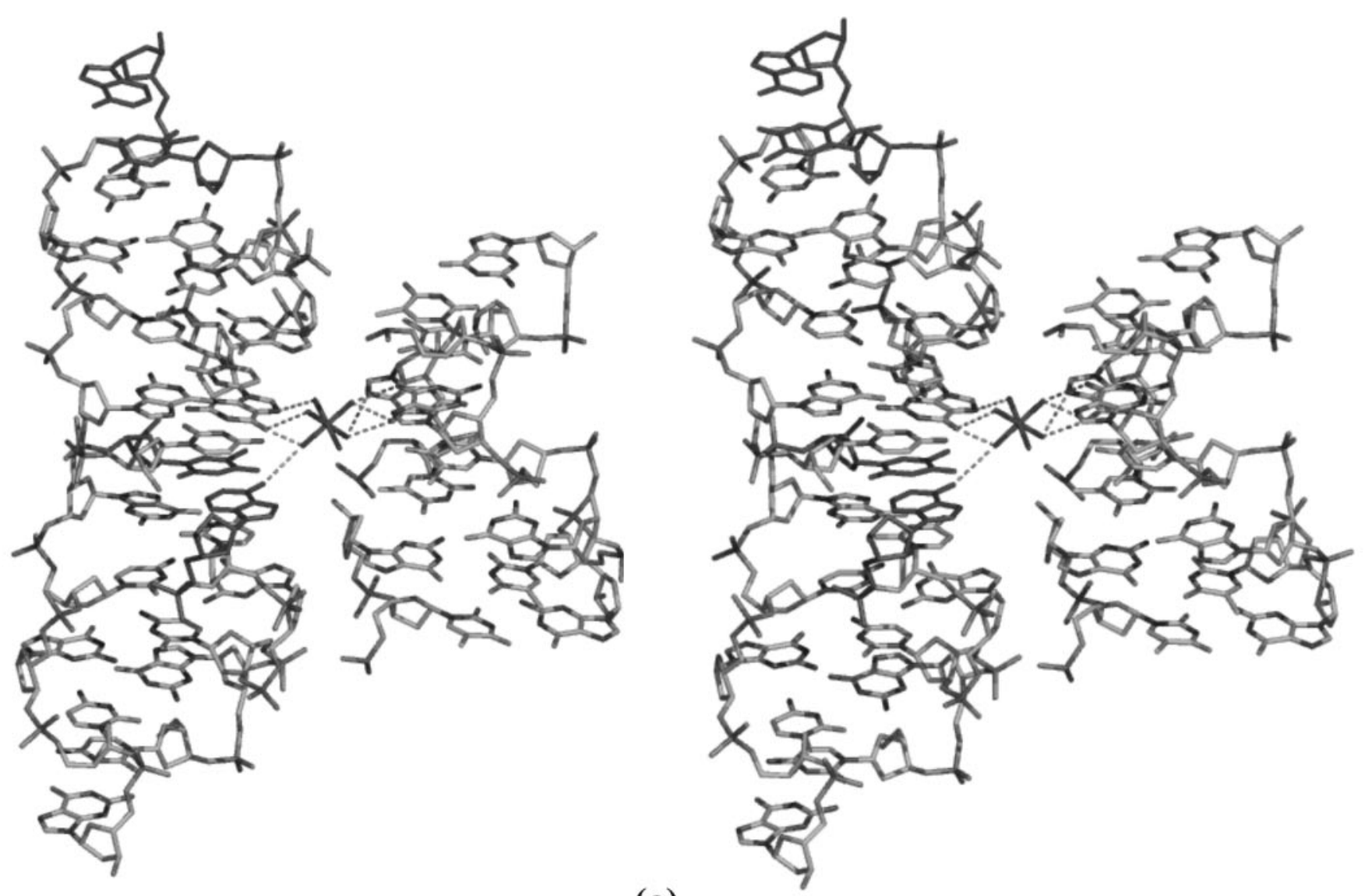

(a)
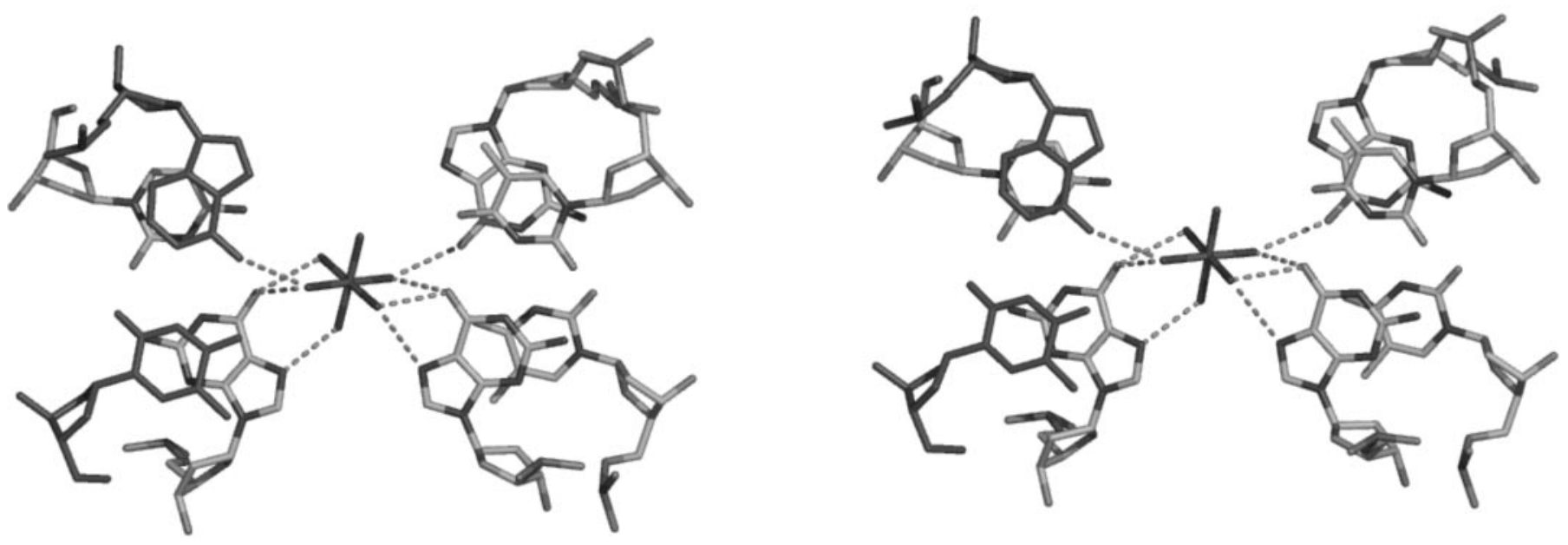

(b)

Figure 4. (a) Stereo view of the ion binding mode in the $\mathrm{P}_{5}$ structure. On the left are two hexamers of HexHA6. On the right is an arbitrary hexamer of DinuHA6. (b) Details of the ion binding mode in the $\mathrm{P}_{5}$ structure.

hexammine ions bind to the adjacent helices such that the helices bridged by the ion are oriented in opposite directions. Thus, along the $a$-axis, the helices are all oriented in the same direction, and there is no ion bridging these helical columns. But along the $b$-axis, the adjacent helical columns are bridged by cobalt hexammine ions, and the alternate columns are aligned in opposite (anti-parallel) directions.

In the hexagonal structure, ordered columns of helices (HexHA6) alternate with disordered columns (DinuHA6) along both axes. The disordered ion binds on one side to 
the G12 residue of HexHA6. On the other side it binds to DinuHA6, which is disordered. Thus the ion binds one-third to adenine and two-thirds to guanine. If we extrapolate the ion binding mode of the orthorhombic structure to this situation, we would expect that, when cobalt hexammine ions bridge the two adjacent columns of helices, these columns should be aligned in opposite directions. However, a lack of a sufficient number of ions results in non-uniformity in orienting these helices. Hence not all the columns of DinuHA6 helices are aligned opposite to the columns of HexHA6 helices. This results in disorder at the positions of DinuHA6 helices; with some columns aligned parallel and others (i.e. those that have ions bound) aligned anti-parallel. These columns are therefore disordered in the crystal, and the 6-fold screw axes coincide with the helical axes of these columns, resulting in the hexagonal space group $\mathrm{P}_{5}$. Hence, this packing mode is probably a direct consequence of insufficient number of ions in the crystallization drop. In other words, we infer that the growth of these crystals is driven by the interactions of the ion with neighbouring helices. When the ratio of ion to DNA in the crystallization drop is $1: 2$, there are just sufficient ions to form crystals with ordered packing, while at lower ratios we obtain disordered crystals.

The binding of the ion has also lead to the formation of a cross-strand hydrogen bond between N6 of adenine and O6 of guanine from a symmetry related helix stacked over it in both the space groups. This could have arisen also due to the stacking interaction between $\mathrm{C}: \mathrm{G}$ and $\mathrm{A}: \mathrm{T}$ base pairs at the virtual step between the two helices. (Hydrogen bonding distances are given in Table 3.) In OrthHA6, the propeller twist at the base pairs involved in the cross-strand bond is $6.71^{\circ}$ for the $\mathrm{C} 1: \mathrm{G} 12$ base pair and $9.24^{\circ}$ for A6:T7 as compared to an average value of $1.4^{\circ}$ for the hexamer, and $-1.34^{\circ}$ in the $\mathrm{Z}$ type helix. In HexHA6, even though the ion bound to G12 is disordered and takes only partial occupancy, the cross-strand hydrogen bond is present. In this case, the tilt of the terminal bases is altered. In HexHA6, the virtual step ApC has a high negative tilt of $-3.6^{\circ}$ as compared to $1.9^{\circ}$ in OrthHA6. Similarly, slide at the virtual step is high in HexHA6, with a value of $\sim-3.6 \AA$, while in OrthHA6 it is only $-0.7 \AA$.

To summarize, the binding of the cobalt hexammine ion to DNA has three distinct effects. First, it induces a tautomeric shift in an adenine base, which leads to the base forming a wobble-type pair with thymine rather than the normal Watson-Crick pair. Second, it binds to both cytosine and guanine of an adjacent GC base pair, and disturbs the base pair geometry. Third, it acts as a strong modulator of tertiary interactions between the DNA helices, leading to disorder and changes in the space group when the ion concentration in lowered. The binding of the ion does not however lead to any significant changes in the $\mathrm{Z}$ type double helical structure of the oligonucleotide duplex.

\section{ACKNOWLEDGEMENTS}

This work was supported by DST-FIST and UGC-SAP funding. S.T. thanks CSIR, India for financial support as SRF. X-ray data collection facilities at the Structural Biology Unit, National Institute of Immunology, New Delhi, GNR Centre for Structural Biology, Central Leather Research Institute,
Chennai and National Area Detector Facility at Molecular Biophysics Unit, Indian Institute of Science, Bangalore are acknowledged.

\section{REFERENCES}

1. Egli,M. (2002) DNA-cation interactions: quo vadis? Chem. Biol., 9, 277-286.

2. Hud,N.V. and Polak,M. (2001) DNA-cation interactions: the major and minor grooves are flexible ionophores. Curr. Opin. Struct. Biol., 11, 293-301.

3. Manning,G.S. (1978) The molecular theory of polyelectrolyte solutions with applications to the electrostatic properties of polynucleotides. Q. Rev. Biophy., 11, 179-246.

4. Holbrook,S.R., Sussman,J.L., Warrant,R.W., Church,G.M. and Kim,S.H. (1977) RNA ligand interactions. (I) Mg binding sites in yeast tRNA ${ }^{\text {Phe }}$. Nucleic Acids Res., 4, 2811-2820.

5. Behe,M.J. and Felsenfeld,G. (1981) Effects of methylation on a synthetic polynucleotide: The B-Z transition in poly $(\mathrm{dG}-\mathrm{m} 5 \mathrm{dC})$.poly $(\mathrm{dG}-\mathrm{m} 5 \mathrm{dC})$. Proc. Natl Acad. Sci. USA, 78, 1619-1623.

6. Rich,A., Nordheim,A. and Wang,A.H.J. (1984) The chemistry and biology of left-handed Z-DNA. Annu. Rev. Biochem., 53, 791-846.

7. Chen,H.H., Behe,M.J. and Rau,D.C. (1984) Critical amount of oligovalent ion binding required for the B-Z transition of poly (dG-m5dC). Nucleic Acids Res., 12, 2381-2389.

8. Klement,R., Soumpasis,D.M. and Jovin,T.M. (1991) Computation of ionic distributions around charged biomolecular structures: results for right-handed and left-handed DNA. Proc. Natl Acad. Sci. USA, 88, 4631-4635.

9. Fenley,M.O., Manning,G.S. and Olson,W.K. (1990) Ionic effects on DNA electrostatic and elastic stability. In Vasilescu,D., Jaz,J., Packer,L. and Pullman,B. (eds), Water and Ions in Biomolecular Systems. Birkhauser Verlag, Basel, Vol. I, pp. 29-44.

10. Demaret,J.P. and Gueron,M. (1993) Composite cylinder models of DNA: application to the B-Z transition of DNA. Biophys. J., 65 , 1700-1713.

11. Gueron,M., Demaret,J.P. and Filoche,M. (2000) A unified theory of the $\mathrm{B}-\mathrm{Z}$ transition of DNA in high and low concentrations of multivalent ions. Biophy. J., 78, 1070-1083.

12. Gessner,R.V., Quigley,G.J., Wang,A.H.J., van der Marel,G.A.,van Boom,J.H. and Rich,A. (1985) Structural basis for stabilization of Z-DNA by cobalt hexammine and magnesium cations. Biochemistry, 24, 237-240.

13. Ho,P.S., Frederick,C.A., Saal,D., Wang,A.H.J. and Rich,A. (1987) The interactions of ruthenium hexammine with Z-DNA: crystal structure of a $\left[\mathrm{Ru}(\mathrm{NH} 3)_{6}\right]^{+3}$ salt of d(CGCGCG) at $1.2 \AA$ resolution. J. Biomol. Struct. Dyn., 4, 521-534.

14. Karthe,P. and Gautham,N. (1998) Structure of $\mathrm{d}(\mathrm{CACGCG}) \mathrm{d}(\mathrm{CGCGTG})$ in crystals grown in the presence of ruthenium (III) hexammine chloride. Acta Crystallogr., D54, 501-509.

15. Sadasivan,C. and Gautham,N. (1995) Sequence-dependent micro heterogeneity of Z-DNA: the crystal and molecular structures of d(CACGCG).d(CGCGTG) and d(CGCACG).d(CGTGCG). J. Mol. Biol., 248, 918-930.

16. Navaza,J. (1994) AMoRe: an automated package for molecular replacement. Acta Crystallogr., A50, 157-163.

17. Collaborative Computational Project, Number 4 (1994) The CCP4 suite: programs for protein crystallography. Acta Crystallogr., D50, 760-763.

18. Wang,A.H.J., Quigley,G.J., Kolpak, van Boom,J.H., van der Marel,G. and Rich,A. (1981) Left-handed double helical DNA: variations in the backbone conformation. Science, 211, 171-176.

19. Murshudov,G.N., Vagin,A.A. and Dodson,E.J. (1997) Refinement of macromolecular structures by the maximum-likelihood method. Acta Crystallogr., D53, 240-255.

20. Dickerson,R.E. (1998) DNA bending: the prevalence of kinkiness and the virtues of normality. Nucleic Acids Res., 26, 1906-1926.

21. Lavery,R. and Sklenar,H. (1988) The definition of generalized helicoidal parameters and of axis curvature for irregular nucleic acids. J. Biomol. Struct. Dyn., 6, 63-91.

22. Ho,P.S. and Mooers,B.H.M. (1997) Z-DNA crystallography. Biopolymers, 44, 65-90. 
23. Hobza,C. and Sandorfy,C. (1985) Perturbation of hydrogen bonds in the adenine $\ldots$ thymine base pair by $\mathrm{Na}^{+}, \mathrm{Mg}^{2+}, \mathrm{Ca}^{2+}$ and $\mathrm{NH}_{4}{ }^{+}$cations. J. Biomol. Struct. Dyn., 2, 1245-1254.

24. Sponer,J., Burda,J.V., Mejzlik,P., Leszczynski,J. and Hobza,P. (1997) Hydrogen-bonded trimers of DNA bases and their interaction with metal cations: $a b$ initio quantum-chemical and empirical potential study. J. Biomol. Struct. Dyn., 14, 613-628.

25. Sponer,J., Leszczynski,J. and Hobza,P. (1996) Hydrogen bonding and stacking of DNA bases: a review of quantum-chemical ab initio studies. J. Biomol. Struct. Dyn., 14, 117-135.

26. Crick,F.H. (1966) Codon-anticodon pairing: the wobble hypothesis. J. Mol. Biol., 19, 548-555.

27. Ho,P.S., Frederick,C.A., Quigley,G.J., van der Marel,G.A., van Boom,J.H., Wang,A.H.J. and Rich,A. (1985) G:T wobble base-pairing in Z-DNA at $1.0 \mathrm{~A}$ atomic resolution: the crystal structure of d(CGCGTG). EMBO J., 4, 3617-3623.

28. Kennard,O. (1985) Structural studies of DNA fragments: the G:T wobble base pair in A, B and Z DNA; the G:A base pair in B-DNA. J. Biomol. Struct. Dyn., 3, 205-226.

29. Cate,J.H., Hanna,R.L. and Doudna,J.A. (1997) A magnesium ion core at the heart of a ribozyme domain. Nature Struct. Biol., 4, 553-558.

30. Cate,J.H. and Doudna,J.A. (1996) Metal-binding sites in the major groove of a large ribozyme domain. Structure, 4, 1221-1229.

31. Cate,J.H., Gooding,A.R., Podell,E., Zhou,K., Golden,B.L., Kundrot,C.E., Cech,T.R. and Doudna,J.A. (1996) RNA tertiary structure mediation by adenosine platforms. Science, 273, 1678-1685.

32. Birnbaum,I.G., Kierdaszuk,B. and Shugar,D. (1984) Tautomerism and conformation of the promutagenic analogue N6-methoxy-2', $3^{\prime}$, 5'-tri-O-methyladenosine. Nucleic Acids Res., 12, 2447-2460.

33. Zamora,F., Kunsman,M., Sabat,M. and Lippert,B. (1997) Metal-stabilized rare tautomers of nucleobases. 6-Imino tautomer of adenine in a mixed-nucleobase complex of mercury (II). Inorg. Chem., 36, 1583-87.

34. Topal,M.D. and Fresco,J.R. (1976) Complementary base pairing and the origin of substitution mutations. Nature, 263, 285-289.

35. Lippert,B. (1997) Effects of metal-ion binding on nucleobase pairing: stabilization, prevention and mismatch formation. J. Chem. Soc., Dalton Trans., 3971-3976.

36. Sigel,H. (1993) Interactions of metal-ions with nucleotides and nucleic-acids and their constituents. Chem. Soc. Rev., 22, 255-267.

37. Gao,Y., Sriram,M. and Wang,A.H.J. (1993) Crystallographic studies of metal ion-DNA interactions: different binding modes of cobalt(II), copper(II) and barium(II) to N7 of guanines in Z-DNA and a drug-DNA complex. Nucleic Acids Res., 17, 4093-4101.

38. Adam,S., Bourtayre,P., Liquier,J. and Taillandier,E. (1986) Interaction of transition metal ions with $\mathrm{Z}$ form poly $\mathrm{d}(\mathrm{A}-\mathrm{C})$.poly $\mathrm{d}(\mathrm{G}-\mathrm{T})$ and poly d(A-T) studied by I.R. spectroscopy. Nucleic Acids Res., 14, 3501-3513. 